\title{
Impact of Canalith Repositioning Maneuver with or Without Vestibular Suppressant Therapy on Anxiety Levels among Patients with Benign Paroxysmal Positional Vertigo
}

(D) Berk Gürpınar, (D) Tolgar Lütfi Kumral, (D) Belgin Tutar, (D) Güler Berkiten, (D) Yavuz Uyar

University of Health Sciences Turkey, Prof. Dr. Cemil Taşçıoğlu City Hospital, Clinic of Otorhinolaryngology, Istanbul, Turkey

\section{Abstract}

Objective: Many studies do not recommend vestibular suppressant as an adjunctive therapy to canalith repositioning maneuver (CRM); nevertheless, it has been proven that those treatment regimens may alter the natural course of treatment by lowering both the anxiety effect and an additive placebo effect. Therefore, we aimed to look for and compare the efficacy of CRM therapy alone with maneuver (M) + vestibular suppressant therapy (M + VST). As a novelty, we used the State-Trait Anxiety Inventory (STAI) to express both the current and underlying anxiety level of the susceptible cases.

Methods: One hundred cases were included in the study and were randomly assigned to either CRM or M + VST groups. Before the therapy, each participant received a 40-item STAI test, and then the select treatment was applied. On the second week, the participants were reevaluated and filled in another STAI.

Results: In CRM, 23 cases were affected from the right side and 27 from the left side. Further, 21 cases received the barbeque $\mathrm{M}$ and 29 the Epley maneuver. On the other hand, in M + VST, 19 cases were affected from the right side and 31 from the left side. In addition, 27 cases received the barbeque $\mathrm{M}$ and 23 the Epley maneuver. The STAI-State (S) and STAI-Trait (T) results were compared before and after the treatment regimens. Pretreatment scores of STAI-S or STAI-T were not significant between the two groups $(p=0.494 ; p=0.481)$. STAI-S and STAI-T scores substantially decreased within the groups after the treatment; in other words, both CRM and M + VST had lower SCores after the treatment regimens $(p<0.05)$. The posttreatment STAI-S and STAI-T scores were lower in $M+V S T$ than that of CRM $(p<0.05)$.

Conclusion: VST is not advised in benign paroxysmal positional vertigo; CRMs alone are proven effective. However, there might be of minimal additional therapeutic effects of drug therapy to control the anxiety levels.

Keywords: Vertigo, maneuver, anxiety

\section{INTRODUCTION}

Vertigo, sensation of whirling and loss of balance, could be experienced by looking down from a high altitude or in symptomatic cases caused by a condition affecting the inner ear. The most common cause of vertigo is benign paroxysmal positional vertigo (BPPV), which causes anxiety in almost every case and is responsible for $20 \%-40 \%$ of cases (1).
This particular subtype of peripheral vertigo is clearly dependent on postural changes, especially the rotation of the head, and is characterized by nystagmus and repetitive short attacks of severe dizziness. Although it has been thought to be linked to psychiatric disorders such as depression, panic attacks, and other anxiety disorders, medically anxiety does not cause vertigo. What it causes is a combination of three different 
experiences mimicking vertigo as a symptom: dizziness, light headedness, and nausea. On the other hand, vertigo triggers anxiety. Information obtained from the inner ear is processed in the amygdala, infralimbic cortex, and hypothalamus which are also a part of the network system for emotional encoding, particularly the parabrachial nucleus (2).

The State-Trait Anxiety Inventory (STAI) is a psychometric scale consisting of 40 statements. STAI has State (S)-Anxiety and Trait (T)Anxiety subscales that evaluate different periods/types of anxiety: STAI-S measures state anxiety and deals with how the respondents feel "right now", using items that measure subjective feelings of apprehension, tension, nervousness, worry, and activation/arousal of the autonomic nervous system, while STAI-T measures the trait anxiety or anxiety level as a part of personal characteristics, including general states of calmness, confidence, and security. The increase in the scores is associated with higher levels of anxiety. The specific attention of all other anxiety questionnaires, other than STAI, is focused on one type of anxiety at the time. However, STAI measures both the current and the trait anxiety (3).

A literature review revealed a study using STAI to measure the anxiety levels of the cases; nevertheless to the best of our knowledge, we found none to compare the anxiety levels after either a canalith repositioning maneuver (CRM) alone or maneuver (M) + vestibular suppressant therapy (VST) (4). It was therefore our intention to carry out a thorough analysis in order to find this specific basis, to contribute to the literature, and thus to assess the efficacy of treatment on anxiety levels more in detail by assessing the STAI subscales.

\section{METHODS}

Between February and April 2020, 100 cases (39 male, 61 female) were included in the study, the approval from the local Ethics Committee of Okmeydanı Research and Education Hospital was taken (04.02.2020/48670771-514.10), and informed consent was obtained from all participants.

All cases underwent a detailed otolaryngologic examination. However, in suspected cases, internal medicine and/or neurological consultations were performed to exclude central or metabolic causes of dizziness. Magnetic resonance imaging scans of the brain and internal acoustic canals and Doppler ultrasounds of the neck were also completed if needed.

Each participant underwent pure tone audiometry (PTA) to detect any disorder through the frequencies between 250 and $8000 \mathrm{~Hz}$ (Interacoustics AC40, Denmark) and completed STAI-S and STAI-T after PTA.
Finally, videonystagmography and video head impulse tests were performed using ICS Impulse video goggles system (GN Otometrics, Schaumburg, IL, USA). The participants were seated in a well-lit room at a distance of $1 \mathrm{~m}$ from a target point on the wall approximately $90 \mathrm{~cm}$ above the floor. A pair of glasses with a high-frequency $(250 \mathrm{~Hz})$ video camera was positioned to record real-time eye movements. There was also a motion sensor to measure head movements. Before each test, calibration was performed to ensure correct measurement. All tests were carried out by a single professional nurse. Moreover, the head impulses were delivered unpredictably. This was required in regard to both direction and timing. The head impulses were applied with a small amplitude $\left(5^{\circ}-20^{\circ}\right)$ and high acceleration $\left(1,000^{\circ} / \mathrm{s}^{2}\right.$ $\left.4,000^{\circ} / \mathrm{s}^{2}\right)$. To conduct a horizontal semicircular canal testing, the hands of the attendant were placed on the jaw and on top of the head. The participant's head was turned $30^{\circ}$ forward in the pitch plane to position the horizontal semicircular canals completely horizontal. Ten to twenty head impulses were delivered to each side. To perform a vertical semicircular canal testing, the hands of the attendant were placed on top of the participant's head, and the fingers were directed toward the anterior semicircular canal to be tested. The chair was rotated $45^{\circ}$ to either side and before vertical semicircular canal testing. In order to test the right anterior (RA) and left posterior (LP) semicircular canal, the chair was turned $45^{\circ}$ to the left, and the participant was asked to look at the fixation dot. For RA, the participant's head was rotated forward in the pitch plane perpendicular to the wall; and for LP, the participant's head was rotated backward in the pitch plane perpendicular to the wall. For the left anterior canal, the participant's head was rotated forward in the pitch plane perpendicular to the wall, whereas for the right posterior canal, the participant's head was rotated backward in the pitch plane perpendicular to the wall. Vestibulo-ocular reflex saccades of the patient were recorded and transferred to a computer. Corrective saccades were defined as transient (fast) eye movements. Those that occur after the head rotation were referred to as overt saccades, whereas those that occur during the head rotation were referred to as covert saccades.

Before proceeding to the vestibular test, all cases were strictly asked not to continue their vestibular suppressant drug therapy for at least 5 days prior to the test. Exclusion criteria included pediatric age group, vision loss, limited neck movement that loses concentration in the test, ongoing vestibular rehabilitation patients, neurological diseases affecting the central nervous system, psychiatric disorders, and being under vestibular suppressant medications. 
Cases were then randomly divided into two groups using a computer-based randomization program (5): CRM and $M$ + VST, both designed as the study group. All cases received the appropriate maneuver in the CRM, whereas in M + VST, maneuver and medication (betahistine dihydrochloride $24 \mathrm{mg}$ $\mathrm{BID}+$ dimenhydrinate $50 \mathrm{mg}$ BID) were both applied for at least 1 month. The control examinations of all cases were done 2 weeks after, and a second STAI-S and STAI-T tests were completed for each case.

\section{Statistical Analysis}

Statistical analysis was performed using IBM SPSS Statistics 22 (IBM SPSS, Turkey) where the continuous data was displayed as the mean \pm standard deviation. The normality of the data distribution was assessed using the Shapiro-Wilk test. Student's t-test was used for between-group comparisons of normally distributed parameters, while the paired sample t-test was applied for in-group comparisons. Moreover, descriptive statistics included mean \pm standard deviations. Statistical significance was considered to be a $p$ value of lesser than 0.05 .

\section{RESULTS}

We evaluated the anxiety scores of 100 cases, equally distributed into two groups: 50 for CRM and 50 for M + VST. The mean age of CRM was $50.84 \pm 12.49$ and the mean age of $\mathrm{M}+$ VST was $36.92 \pm 10.24$. Furthermore, the age comparison between the groups was not significant ( $p>0.05)$. In the CRM group, 16 (41.0\%) were male and 23 (59.0\%) were female, whereas in $\mathrm{M}+$ VST group, 34 (55.7\%) were male and 27 (44.3\%) were female. Likewise, gender between the groups was also not significant $(p>0.05)$ (Table 1).

In CRM, 23 cases were affected from the right side and 27 from the left side (27 posterior, 21 lateral, and 2 superior semicircular canal pathologies). Twenty-one cases received the barbeque maneuver and 29 received the Epley maneuver. On the other hand, in M + VST, 19 cases were affected from the right side and 31 from the left side ( 26 posterior, 23 lateral, and 1 superior semicircular canal pathology). Twenty-six cases

\begin{tabular}{|c|c|c|c|}
\hline & CRM $(n=50)$ & $M+\operatorname{VST}(n=50)$ & $\mathbf{p}$ \\
\hline Age (Mean \pm SD) & $40.84 \pm 12.49$ & $36.92 \pm 10.24$ & 0.122 \\
\hline \multicolumn{4}{|l|}{ Gender (n, \%) } \\
\hline Male & $16(41.0 \%)$ & $34(55.7 \%)$ & \multirow{2}{*}{0.219} \\
\hline Female & $23(59.0 \%)$ & $27(44.3 \%)$ & \\
\hline \multicolumn{4}{|c|}{$\begin{array}{l}\text { Student's t-test for age, continuity (yates) correction for gender is applied, } \mathrm{p}<0.01 \\
\text { CRM: Canalith repositioning maneuver-only group, M + VST: Vestibular suppressan } \\
\text { therapy + canalith repositioning maneuver group, SD: Standard deviation }\end{array}$} \\
\hline
\end{tabular}

received the barbeque maneuver and 24 received the Epley maneuver.

The STAI-S and STAI-T results were compared before and after the treatment regimens. Pretreatment scores of STAI-T were not significant between the two groups ( $p=0.692$ ). STAI-S levels in both groups significantly decreased after adequate therapy $(p=0.001 ; p=0.001)$. STAI-S and STAI-T scores substantially decreased within the groups after the treatment, i.e., both CRM and $M+$ VST had lower scores after the treatment regimens $(p=0.001)$. The posttreatment STAI-S and STAI-T scores were lower in M + VST than that of CRM and were statistically significant $(p=0.001)$ (Table 2).

\section{DISCUSSION}

Anxiety and fear have long been recognized by the clinicians to be associated with vestibular symptoms, namely, loss of balance, dizziness, or nausea, are perceived as frightening, catastrophic, or deadly. Therefore, it is usually imperative to investigate the interface between anxiety and vestibular disturbance (2).

Majority of the vestibular disorders are BPPV, and the mainstay of therapy in BPPV is the canal repositioning maneuver. A single maneuver is effective as high as 70\%-90\%, and patients are often free from symptoms after a single maneuver. Some additional measures, such as sedation, head vibration during treatment, keeping the head in an upright position for 48 hours to prevent the particles from entering the canal, avoiding lying down with the affected ear for 1 week, wearing a neck collar, and avoiding a positional test examination for about a week after treatment, have been proposed to improve the effectiveness of the treatment $(6,7)$.

Table 2. Intergroup and intragroup comparisons for STAI-T and STAI-S

\begin{tabular}{|c|c|c|c|c|}
\hline & & $\begin{array}{l}\text { CRM } \\
(n=50)\end{array}$ & $\begin{array}{l}M+V S T \\
(n=50)\end{array}$ & $1 p$ \\
\hline \multirow{4}{*}{ STAI-S } & Pretreatment & $64.02 \pm 6.89$ & $66.88 \pm 5.44$ & $0.023^{*}$ \\
\hline & Posttreatment & $32.24 \pm 4.15$ & $23.88 \pm 2.51$ & $0.001^{*}$ \\
\hline & Difference (\%) & $31.78 \pm 7.60$ & $43.01 \pm 6.11$ & $0.001^{*}$ \\
\hline & $2 p$ & 0.001 & 0.001 & \\
\hline \multirow{4}{*}{ STAI-T } & Pretreatment & $67.98 \pm 7.88$ & $68.54 \pm 6.07$ & 0.692 \\
\hline & Posttreatment & $34.18 \pm 4.10$ & $24.36 \pm 2.68$ & $0.001^{*}$ \\
\hline & Difference (\%) & $33.80 \pm 8.31$ & $44.18 \pm 6.81$ & $0.001 *$ \\
\hline & $2 p$ & 0.001 & 0.001 & \\
\hline
\end{tabular}


A recent study included 64 cases diagnosed with BPPV. These cases were then grouped into three. In the first group, only CRM was performed, while in the second group, CRM with betahistine $24 \mathrm{mg}$ twice daily was administered for 10 days. In the third group, CRM with dimenhydrinate $50 \mathrm{mg}$ once daily was given for 5 days. Cases were reexamined on the $10^{\text {th }}$ day, showing no superiority of one group over another, suggesting that drugs were no beneficial in the BPPV (8). However, we have not achieved results in accordance with the abovementioned article. The anxiety levels of M + VST was significantly lower than that of the CRM group. Nevertheless, in the clinical practice guideline for BPPV, VST was not routinely recommended for its side effects, such as drowsiness that impedes social life, but it was found helpful in treating symptoms such as nausea or vomiting in severely symptomatic cases. The guideline also advices not to use them in severely symptomatic cases that refuse therapy or become severely symptomatic after a positioning maneuver (9).

Various studies have reported concurrent psychiatric symptoms in some BPPV cases $(10,11)$. The percentage of concurrence is thought to be greater among patients with psychiatric disorders. Moreover, cases that have comorbid psychiatric symptoms may have dizziness even in the absence of objective tests. Those cases refrain themselves from social interactions, and their anxiety level is thought to be more than the healthy population (12). This was the limitation of our study, where psychiatric disorders are excluded.

Published data revealed that vertigo patients had anxiety at a rate between 30\% and 73.5\%. These reports have used several different methods to identify the level of anxiety such as Hospital Anxiety and Depression scale, STAI, or Beck Anxiety Inventory. Regardless of the different scales that measure anxiety, the anxiety levels were proven higher than that of anxiety disorders in the overall population (13-16). In our study, all of our cases had severe anxiety prior to treatment, but the level of anxiety decreased too much extent 2 weeks after the ongoing treatment.

Recently, owing to the effects of placebo on improved self-control, comprehensive studies have shown the effects of placebo on neuralgia, psychiatric disorders, and neurological diseases such as Parkinson's disease $(17,18)$. These studies showed that the beliefs altered the neural processes and changed the perception and emotion. Although our study was not based on a placebo effect, considering the aforementioned reports and guidelines on the excessive use of drugs in BPPV, our results, on the contrary, have proven that drug therapy administered concurrently with the CRM has helped to relieve anxiety to a much lower extent.

\section{CONCLUSION}

VST is not recommended in BPPV; however, CRMs alone are proven effective. Nevertheless, in symptomatic cases, a shortterm use of suppressant therapy has been proven effective in controlling anxiety levels.

\section{Ethics}

Ethics Committee Approval: Ethics Committee of Okmeydanı Research and Education Hospital was taken (04.02.2020/48670771-514.10).

Informed Consent: Informed consent was obtained from all participants.

Peer-review: Externally and internally peer-reviewed.

\section{Authorship Contributions}

Surgical and Medical Practices: G.B., Concept: B.G., G.B., Design: B.G., B.T., Data Collection or Processing: T.L.K., Analysis or Interpretation: T.L.K., Y.U., Literature Search: B.T., Writing: B.G., G.B., Y.U.

Conflict of Interest: No conflict of interest was declared by the authors.

Financial Disclosure: The authors declared that this study received no financial support.

\section{REFERENCES}

1. Tang $\mathrm{H}$, Li W. Advances in the diagnosis and treatment of benign paroxysmal positional vertigo. Exp Ther Med 2017;14:2424-30.

2. Hagr A. Comorbid psychiatric conditions of benign paroxysmal positional vertigo. Int J Health Sci (Qassim) 2009;3:23-8.

3. Spielberger CD, Sydeman SJ. State-Trait Anxiety Inventory and State-Trait Anger Expression Inventory. In: The and outcome assessment. Mark Edward, editors. Hillsdale, NJ: Lawrence Erlbaum Associates; 1994 p. 292-321.

4. Ferrari S, Monzani D, Baraldi S, Simoni E, Prati G, Forghieri M, et al. Vertigo "in the pink": The impact of female gender on psychiatricpsychosomatic comorbidity in benign paroxysmal positional vertigo patients. Psychosomatics 2014;55:280-8.

5. Urbaniak GC, Plous S. Research Randomizer (Version 4.0) [Computer software]. Last accessed date: 22.06.2013. Available from: http://www. randomizer.org/

6. Epley JM. The canalith repositioning procedure for treatment of benign paroxysmal positional vertigo. Otolaryngol Head Neck Surg 1992;107:399-404.

7. Semont A, Freyss G, Vitte E. Curing the BPPV with a liberatory maneuver. Adv Oto-Rhino-Laryng 1988;42:290-3.

8. Inan HC, Kıraç M. An Evaluation of the Effects of betahistine and dimenhydrinate on posterior canal benign paroxysmal positional vertigo. Turk Arch Otorhinolaryngol 2019;57:191-6. 
9. Bhattacharyya N, Gubbels SP, Schwartz SR, Edlow JA, El-Kashlan H, Fife T, et al. Clinical practice guideline: benign paroxysmal positional vertigo (update). Otoolaryngol Head Neck Surg 2017;156:S1-47.

10. Eagger S, Luxon L, Davies R, Coelho A, Ron M. Psychiatric morbidity in patients with peripheral vestibular disorder: a clinical and neurootological study. J Neurol Neurosurg Psychiatry 1992;55:383-7.

11. Mckenna L, Hallam RS, Hinchcliffef R. The prevalence of psychological disturbance in neuro-otology outpatients. Clin Otolaryngol Allied Sci 1991;16:452-6.

12. Yardley L. Overview of psychologic effects of chronic dizziness and balance disorders. Otolaryngol Clin North Am 2000;33:603-16.

13. Yardley L, Verschuur C, Masson E, Luxon L, Haacke N. Somatic and psychological factors contributing to handicap in people with vertigo. $\mathrm{Br}$ J Audiol 1992;26:283-90.
14. Yardley L, Luxon L, Haacke NP. A longitudinal study of symptoms, anxiety and subjective well-being in patients with vertigo. Clin Otolaryngol Allied Sci 1994;19:109-16.

15. Nagarkar AN, Gupta AK, Mann SBS. Psychological findings in benign paroxysmal positional vertigo and psychogenic vertigo. J Otolaryngol 2000;29:154-8.

16. Monzani D, Genovese E, Rovatti V, Ludovica Malagoli M, Rigatelli M, et al. Life events and benign paroxysmal positional vertigo: a case-controlled study. Acta Otolaryngol 2006;126:987-92.

17. Pollo A, Torre E, Lopiano L, Rizzone M, Lanotte M, Cavanna A, et al. Expectation modulates the response to subthalamic nucleus stimulation in Parkinsonian patients. Neuroreport 2002;13:1383-6.

18. Phan KL, Fitzgerald DA, Nathan PJ, Moore GJ, Uhde TW, Tancer ME. Neural substrates for voluntary suppression of negative affect: a functional magnetic resonance imaging study. Biol Psychiatry 2005;57:210-9. 